\title{
52. CARBON AND CARBONATE ANALYSES, LEG 34
}

\author{
Donald H. Cameron, Scripps Institution of Oceanography, La Jolla, California
}

Leg 34 sediments were analyzed for total carbon and acid-insoluble (organic) carbon using a LECO 70second analyzer. Detailed descriptions of the technique and theory may be found in Baker, Gerard, et al. (1970) and Boyce and Bode (1972). For control purposes a standard sediment was made up from Deep Sea Drilling material and analyzed for total carbon at predetermined intervals with regular samples. Listed below is the statistical data for this standard.

\begin{tabular}{ccccc}
\hline $\begin{array}{c}\text { DSDP } \\
\text { Standard }\end{array}$ & $\begin{array}{c}\text { No. of } \\
\text { Samples }\end{array}$ & $\begin{array}{c}\text { Total } \\
\text { Carbon as \% } \\
\text { of } \mathrm{CaCO}_{3}\end{array}$ & $\begin{array}{c}\text { Standard } \\
\text { Deviation }\end{array}$ & $\begin{array}{c}\text { Maximum } \\
\text { Range }\end{array}$ \\
\hline 2 & 7 & 80.3 & $0.3 \%$ & $0.6 \%$ \\
\hline
\end{tabular}

Although the number of standards run was small, the results compare very favorably with those run during analyses of samples from previous legs (see Bode, 1974).

Carbon and carbonate data presented in Table 1 are given in weight percent.

\section{REFERENCES}

Bader, R.G., Gerard, R.D., et al., 1970. Initial Reports of the Deep Sea Drilling Project, Volume 4: Washington (U.S. Government Printing Office).

Bode, G.W., 1974. Carbon and carbonate analyses, Leg 27, Deep Sea Drilling Project. In Veevers, J., Heirtzler, J. R., et al., Initial Reports of the Deep Sea Drilling Project, Volume 27: Washington (U.S. Government Printing Office), p. 499.

Boyce, R.E. and Bode, G.W., 1972. Carbon and carbonate analyses, Leg 9, Deep Sea Drilling Project. In Hayes, J.D., et al., Initial Reports of the Deep Sea Drilling Project, Volume 9: Washington (U.S. Government Printing Office), p. 747.

TABLE 1

Carbon and Carbonate Analyses, Leg 34

\begin{tabular}{lcccc}
\hline $\begin{array}{c}\text { Sample } \\
\text { (Interval in cm) }\end{array}$ & $\begin{array}{c}\text { Subbottom } \\
\text { Depth } \\
(\mathrm{m})\end{array}$ & $\begin{array}{c}\text { Total } \\
\text { Carbon } \\
(\%)\end{array}$ & $\begin{array}{c}\text { Organic } \\
\text { Carbon }\end{array}$ & $\mathrm{CaCO}_{3}$ \\
\hline Hole 319 & & & & \\
$1-2,20$ & 2.0 & 0.2 & 0.1 & 0 \\
$1-2,40$ & 2.2 & 0.1 & 0.2 & 0 \\
$1-3,30$ & 3.6 & 0.1 & 0.2 & 0 \\
$1-4,87$ & 5.6 & 4.6 & 0.1 & 37 \\
$1-5,20$ & 6.5 & 6.7 & 0.1 & 55 \\
$1-6,60$ & 8.4 & 0.4 & 0.2 & 2 \\
$2-1,148$ & 11.0 & 7.3 & 0.1 & 60 \\
$2-2,12$ & 11.1 & 7.9 & 0.1 & 65 \\
$2-3,10$ & 12.6 & 7.7 & 0.1 & 63 \\
$2-4,19$ & 14.2 & 7.0 & 0.1 & 58 \\
$3-2.10$ & 20.6 & 8.0 & 0.1 & 66 \\
$3-3,45$ & 22.5 & 9.8 & 0.1 & 81 \\
$4-1,39$ & 29.1 & 9.7 & 0.1 & 80 \\
$4-2,9$ & 30.3 & 9.7 & 0.1 & 80 \\
$4-3,6$ & 31.8 & 10.3 & 0.1 & 85 \\
\hline
\end{tabular}

TABLE 1 - Continued

\begin{tabular}{|c|c|c|c|c|}
\hline $\begin{array}{c}\text { Sample } \\
\text { (Interval in } \mathrm{cm} \text { ) }\end{array}$ & $\begin{array}{l}\text { Subbottom } \\
\text { Depth } \\
\text { (m) }\end{array}$ & $\begin{array}{c}\text { Total } \\
\text { Carbon } \\
(\%)\end{array}$ & $\begin{array}{l}\text { Organic } \\
\text { Carbon }\end{array}$ & $\mathrm{CaCO}_{3}$ \\
\hline $4-4,10$ & 33.3 & 10.0 & 0.1 & 83 \\
\hline $4-5,15$ & 34.9 & 10.8 & 0.1 & 89 \\
\hline $4-6,20$ & 36.4 & 11.2 & 0.1 & 93 \\
\hline $5-2,80$ & 40.3 & 11.5 & 0.0 & 95 \\
\hline $5-3,20$ & 41.2 & 11.4 & 0.0 & 95 \\
\hline $5-4,20$ & 42.7 & 11.5 & 0.1 & 95 \\
\hline $5-5,20$ & 44.2 & 11.5 & 0.1 & 95 \\
\hline $5-6,20$ & 45.7 & 11.5 & 0.1 & 95 \\
\hline $6-1,123$ & 48.7 & 11.5 & 0.1 & 95 \\
\hline $6-3,119$ & 51.7 & 11.5 & 0.0 & 96 \\
\hline $6-4,17$ & 52.2 & 11.5 & 0.0 & 95 \\
\hline $6-5,131$ & 54.8 & 11.3 & 0.0 & 94 \\
\hline $6-6,143$ & 56.4 & 11.5 & 0.0 & 95 \\
\hline $7-1,91$ & 58.1 & 11.2 & 0.1 & 93 \\
\hline $7-2,21$ & 58.9 & 11.5 & 0.0 & 95 \\
\hline $7-3,31$ & 60.5 & 11.5 & 0.1 & 95 \\
\hline $7-4,45$ & 62.2 & 11.4 & 0.0 & 94 \\
\hline $7-5,137$ & 64.6 & 11.4 & 0.0 & 95 \\
\hline $7-6,30$ & 65.0 & 11.3 & 0.1 & 94 \\
\hline $8-1,85$ & 67.4 & 11.3 & 0.1 & 93 \\
\hline $8-2,68$ & 68.7 & 11.0 & 0.1 & 91 \\
\hline $8-4,125$ & 72.3 & 11.3 & 0.1 & 93 \\
\hline $8-5,100$ & 73.5 & 11.0 & 0.1 & 92 \\
\hline $8-6,67$ & 74.7 & 11.5 & 0.1 & 95 \\
\hline $9-1,110$ & 77.1 & 11.0 & 0.1 & 91 \\
\hline $9-2,130$ & 78.8 & 9.2 & 0.1 & 76 \\
\hline $9-3,103$ & 80.0 & 10.3 & 0.1 & 86 \\
\hline $10-1,127$ & 86.8 & 10.8 & 0.1 & 89 \\
\hline $10-2,63$ & 87.6 & 10.9 & 0.1 & 90 \\
\hline $10-3,40$ & 88.9 & 11.2 & 0.1 & 93 \\
\hline $10-4,112$ & 91.1 & 11.1 & 0.1 & 92 \\
\hline $10-5,108$ & 92.6 & 10.7 & 0.1 & 89 \\
\hline $11-1,50$ & 95.7 & 11.0 & 0.0 & 91 \\
\hline $11-2,71$ & 97.4 & 9.9 & 0.0 & 82 \\
\hline $11-3,100$ & 99.2 & 9.3 & 0.1 & 77 \\
\hline $11-4,90$ & 100.6 & 10.4 & 0.0 & 86 \\
\hline $11-5,63$ & 101.8 & 10.4 & 0.1 & 86 \\
\hline $11-6,90$ & 103.6 & 10.8 & 0.0 & 90 \\
\hline $12-1,142$ & 105.9 & 10.9 & 0.1 & 90 \\
\hline $12-2,85$ & 106.9 & 11.0 & 0.1 & 91 \\
\hline $12-3,87$ & 108.4 & 11.3 & 0.1 & 93 \\
\hline
\end{tabular}

Hole 320

$1-1,50$

$1-2,11$

$1-3,7$

$1-4,39$

$1-5,120$

$1-6,51$

$2-1,106$

$2-2,140$

$3-1,84$

$3-2,81$

$3-3,81$

$3-4,57$

$3-5,106$

$3-6,52$

6.8
7.9
9.4
11.2
13.5
14.3
74.6
76.4
103.3
104.8
106.3
107.6
109.6
110.5

$\begin{array}{rrr}1.1 & 1.1 & 0 \\ 1.8 & 1.4 & 3 \\ 1.2 & 1.2 & 1 \\ 0.5 & 0.5 & 0 \\ 1.4 & 1.3 & 1 \\ 1.4 & 1.2 & 2 \\ 7.7 & 0.1 & 63 \\ 7.3 & 0.1 & 60 \\ 11.1 & 0.1 & 92 \\ 11.0 & 0.1 & 91 \\ 11.0 & 0.1 & 91 \\ 11.1 & 0.1 & 92 \\ 11.0 & 0.1 & 91 \\ 10.7 & 0.1 & 89\end{array}$


TABLE 1 - Continued

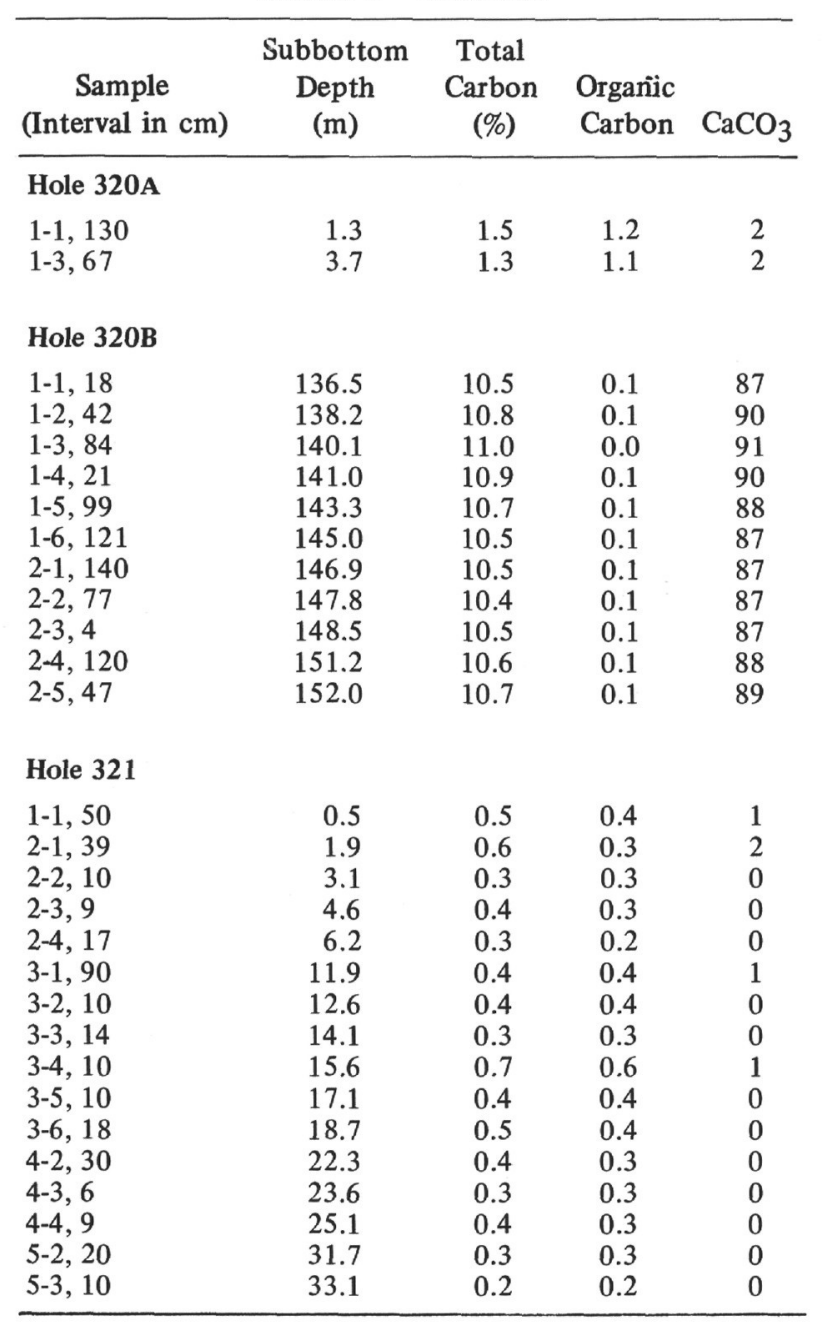

TABLE 1 - Continued

\begin{tabular}{|c|c|c|c|c|}
\hline $\begin{array}{c}\text { Sample } \\
(\text { Interval in } \mathrm{cm})\end{array}$ & $\begin{array}{l}\text { Subbottom } \\
\text { Depth } \\
\text { (m) }\end{array}$ & $\begin{array}{c}\text { Total } \\
\text { Carbon } \\
(\%)\end{array}$ & $\begin{array}{l}\text { Organic } \\
\text { Carbon }\end{array}$ & $\mathrm{CaCO}_{3}$ \\
\hline $5-4,4$ & 34.5 & 0.3 & 0.3 & 0 \\
\hline $5-5,9$ & 36.1 & 0.2 & 0.2 & 0 \\
\hline $5-6,10$ & 37.6 & 0.2 & 0.2 & 0 \\
\hline $6-2,142$ & 42.4 & 0.3 & 0.2 & 1 \\
\hline $6-3,4$ & 42.5 & 0.4 & 0.2 & 2 \\
\hline $6-4,7$ & 44.1 & 0.2 & 0.2 & 0 \\
\hline $6-5,6$ & 45.6 & 0.3 & 0.2 & 1 \\
\hline $6-6,9$ & 47.1 & 0.2 & 0.2 & 0 \\
\hline $7-2,35$ & 50.9 & 0.1 & 0.1 & 0 \\
\hline $7-1,7$ & 52.1 & 0.1 & 0.1 & 0 \\
\hline $7-4,8$ & 53.6 & 0.2 & 0.1 & 0 \\
\hline $7-5,2$ & 55.0 & 0.2 & 0.1 & 0 \\
\hline $7-6,6$ & 56.6 & 0.2 & 0.1 & 0 \\
\hline $7-6,147$ & 58.0 & 10.7 & 0.0 & 89 \\
\hline $8-1,30$ & 58.8 & 11.4 & 0.0 & 95 \\
\hline $8-2,14$ & 60.1 & 11.4 & 0.1 & 95 \\
\hline $8-3,12$ & 61.6 & 11.4 & 0.0 & 94 \\
\hline $8-4,33$ & 63.3 & 11.4 & 0.0 & 95 \\
\hline $8-5,135$ & 65.9 & 11.2 & 0.0 & 93 \\
\hline $8-6,15$ & 66.2 & 11.4 & 0.0 & 95 \\
\hline $9-1,98$ & 69.2 & 6.8 & 0.1 & 56 \\
\hline $9-2,112$ & 70.8 & 9.2 & 0.1 & 76 \\
\hline $9-3,65$ & 71.9 & 9.9 & 0.1 & 82 \\
\hline $9-4,66$ & 73.4 & 9.0 & 0.1 & 74 \\
\hline $9-5,4$ & 74.2 & 9.5 & 0.1 & 78 \\
\hline $9-6,49$ & 76.2 & 6.4 & 0.1 & 52 \\
\hline $10-1,100$ & 78.5 & 10.8 & 0.1 & 90 \\
\hline $10-2,10$ & 79.1 & 11.0 & 0.1 & 91 \\
\hline $10-3,10$ & 80.6 & 11.1 & 0.1 & 92 \\
\hline $11-1,48$ & 87.5 & 11.2 & 0.1 & 93 \\
\hline $11-2,10$ & 88.6 & 11.2 & 0.1 & 93 \\
\hline $11-3,10$ & 90.1 & 11.1 & 0.1 & 92 \\
\hline $11-4,7$ & 91.6 & 11.0 & 0.1 & 91 \\
\hline $13-1,88$ & 116.4 & 10.4 & 0.1 & 86 \\
\hline $13-2,14$ & 117.1 & 10.1 & 0.1 & 84 \\
\hline $13-3,10$ & 118.6 & 9.8 & 0.1 & 81 \\
\hline
\end{tabular}

